\title{
Rapid Soft Lithography by Bottom-Up Enhanced Capillarity
}

\author{
Dario Pisignano,* Francesca Di Benedetto, Luana Persano, Giuseppe Gigli, and \\ Roberto Cingolani
}

NNL, National Nanotechnology Laboratory of Istituto Nazionale di Fisica dela Materia (INFM), cl o Dipartimento di Ingegneria del'Innovazione, Università di Lecce, via Arnesano, I-73100 Lecce, Italy

Received J anuary 7, 2004

\begin{abstract}
The growing demand for new solutions to pursue the trend of micro- and nanoelectronics predicted by Moore's law is stimulating the development of new high-resolution, low-cost lithographies. Here we demonstratethat several bottom-up approaches can be used to increase thethroughput of soft lithography by exploiting the enhanced hydrophilicity, the low viscosity, and the fragility of the employed materials. In particular, thecustomized functional ization of theinvolved surfaces to improvethewettability to polymer fluids and the dramatic decrease of the viscosity of polymer compounds as the temperature is increased, together with the good thermal stability of the functionalized surfaces, allow a faster filling of elastomeric channels, up to almost an order of magnitude with respect to conventional microfluidics.
\end{abstract}

Soft lithography ${ }^{1}$ offers an inexpensive and flexible approach to micro- and nanofabrication for both bioorganic laboratories and massive semiconductor production. Being based on the realization of elastomeric el ements from a master template, soft lithography all ows the pattern transfer through the conformal contact between the replicas and the target material. Soft lithography does not use high-energy beam or organic solvents. It is mechanical, thus ensuring the largest flexibility in terms of employable compounds, low-cost throughput, and high (sub-micrometer) resolution. For these reasons, soft lithography has become the most appealing candidatefor various applications such as lowcost electronics, patterning of bio-organic matter, biodiagnostics, sensors, and lab-on-chips.

Among other mechanical lithographies, such as nanoimprinting, ${ }^{2}$ soft molding, ${ }^{3}$ and microcontact printing, ${ }^{4}$ particular interest has been raised by microfluidic lithography or micromol ding in capillaries (MIMIC), ${ }^{5}$ whose operation principle is schematized in Figure 1a. This method is based on the capillarity-induced filling of el astomeric channels by a polymer or by a suitably diluted polymer solution. In this way, MI MIC allows the realization of polymeric masks to be used as the resist pattern in the following, top-down pattern transfer by wet or reactive ion etching. It is an additive microfabrication technique, using extremely small quantities of materials without wastes. Sofar, therealization of silicon ${ }^{6}$ and fully organic ${ }^{7}$ field effect transistors, optically amplified polymeric waveguides, 8,9 and directional carbon nanotube strings ${ }^{10}$ has been demonstrated by MIMIC. In addition, it is themost suitablemethod for patterning biomolecules and for the selective delivery of proteins from aqueous

* To whom correspondence may be addressed. E-mail: dario.pisignano@unile.it.

(1) Xia, Y.; Whitesides, G. M. Angew. Chem., Int. Ed. Engl. 1998, 37, 550-575.

(2) Chou, S. Y.: Krauss, P. R.; Renstrom, P. J . Science 1996, 272 85-87.

(3) Suh, K. Y.; Kim, Y. S.; Lee, H. H. Adv. Mater. 2001, 13, 13861389.

(4) Kumar, A.; Whitesides, G. M. Appl. Phys. Lett. 1993, 63, 20022004.

(5) Kim, E.; Xia, Y.; Whitesides, G. M. Nature 1995, 376, 581-584.

(6) J eon, N. L.; Hu, J .; Whitesides, G. M.; Erhardt, M. K.; Nuzzo, R. G. Adv. Mater. 1998, 17, 1466-1469.

(7) Beh, W. S.; Kim, I. T.; Qin, D.; Xia, Y.; Whitesides, G. M. Adv. Mater. 1999, 11, 1038-1041. (a)
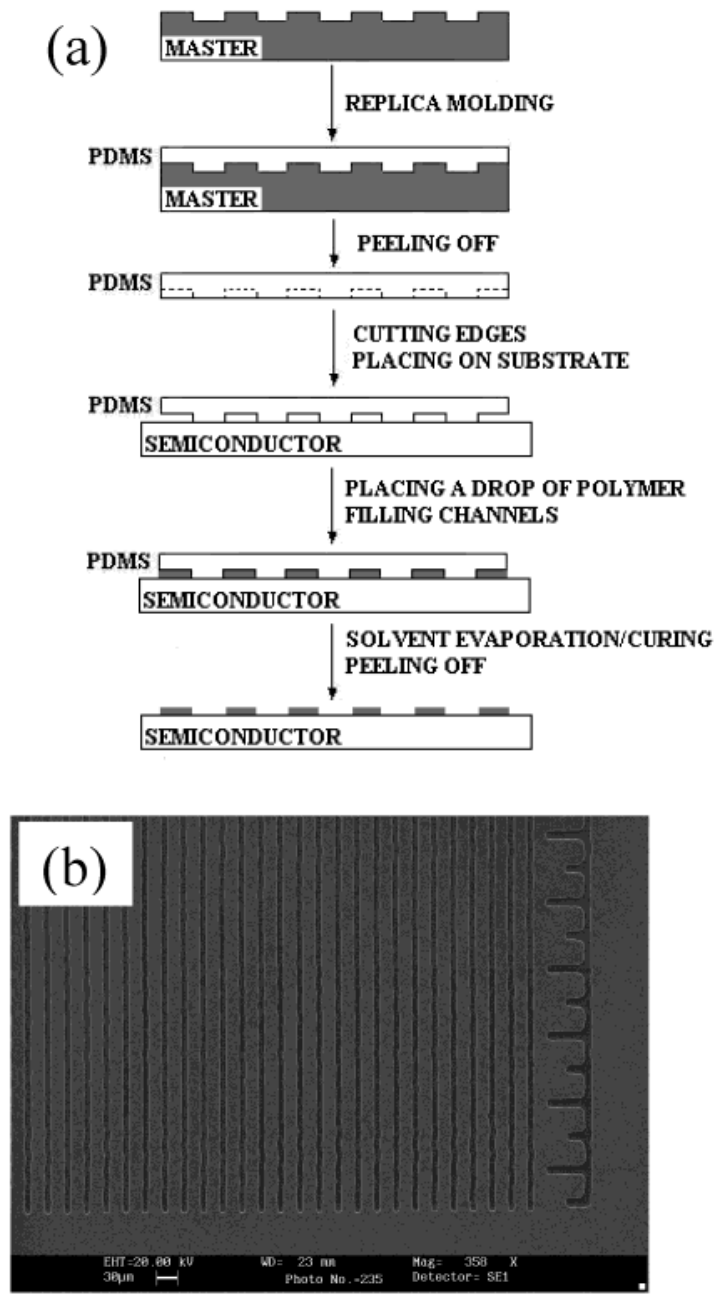

Figure 1. (a) Scheme of the basic MIMIC procedure (features not in scale). (b) E mployed master, showing theintegrated ruler parallel to the capillary $\mu$-channels.

solutions, ${ }^{11-13}$ which is the origin for a worldwideinterest for applying MIMIC to DNA chips and proteomics.

Despitetheseadvantages, a critical drawback of MIMIC, which strongly limits its use for large area applications, is theintrinsicslowness in the pattern formation. Previous 


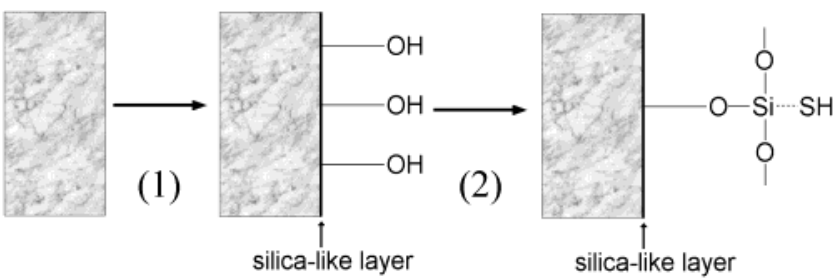

Figure 2. Step 1: PDMSOX was obtained by a few seconds of $\mathrm{O}_{2}$ plasma. Step 2: Incubation of PDMSOX into 3-MPS ethanol solutions resulted in PDMS ${ }^{\mathrm{SH}}$. Si supports were hydroxylated by immersion in freshly prepared piranha solutions for $90 \mathrm{~min}$ and thoroughly rinsed three times for $5 \mathrm{~min}$ in bidistilled and deionized water under ultrasonication at room temperature.

studies increasing the filling speed by vacuum-assisted capillarity ${ }^{14}$ required a quite compl ex setup and resulted in a limited resolution duetothecollapseof theelastomeric elements under vacuum.

Our approach is to selectively adjust the physical parameters responsible for the MIMIC filling rate. The filling rate of the fluidic channels is in fact proportional to the reciprocal length coordinate of the capillary, $z::^{15}$ $\mathrm{dz} / \mathrm{dt}=\mathrm{R} \gamma \cos \Theta / 4 \eta \mathrm{z}$, where $\eta, \mathrm{R}, \gamma$, and $\Theta$ indicate the liquid viscosity, the radius of the capillary, the liquidvapor interfacial free energy of the fluid, and the contact angle between the liquid and the surface of the capillary, respectively. $\Theta$, whose cosine is proportional to the difference between the solid-liquid and the solid-vapor interfacial energies, determines whether thefilling process is ther modynamically favored, i.e., if the channels can be wetted by the polymer $(\cos \Theta>0)$ or $\operatorname{not}(\cos \Theta<0)$. To improve the lithography performances, besides the geometrical factors ( $R$ and $z$ ) affecting the shape of the channels, i.e., the achievable resolution and patterned areas, one can modify $\Theta$ by suitable functionalizations of the involved surfaces, thus increasing their wettability. In our investigation we employed polyurethane, as the polymeric fluid, and gratings consisting of about $11 \mu \mathrm{m}$ wide parallel stripes of period $30 \mu \mathrm{m}$, as the molds. A ruler was integrated in the masters, side by side with the channels, to exactly determine by optical microscopy the length covered by the polymer fluid during the filling process (F igure 1b). With our geometry, the polymer took about $6400 \mathrm{~s}$ to fill a $1 \mathrm{~cm}$ long channel in an untreated silicon sample (open circles in Figure 3a).

Since the filling polyurethane liquid is hydrophilic, ${ }^{16}$ the lithography performances can be improved by customizing the capillary surfaces (i.e., $\Theta$ ) by proper polar moieties. Wefirst covered thewalls of our $\mu$-channels with $-\mathrm{OH}$ functionalities, hydroxilating the Si surfaces by immersion in piranha solution and exposing the patterned side of PDMS to an $\mathrm{O}_{2}$ plasma treatment ${ }^{17-19}$ (step 1 in Figure 2). The corresponding experimental $(z, t)$ curves

(8) Zhao, X.-M.; Stoddart, A.; Smith, S. P.; Kim, E.; Xia, Y.; Prentiss, M.; Whitesides, G. M. Adv. Mater. 1996, 8, 420 .

(9) Yang, P.; Wirnsberger, G.; Huang, H. C.; Corsero, S. R.; McGehee, M. D.; Scott, B.; Deng, T.; Whitesides, G. M.; Chmelka, B. F.; Buratto, S. K.; Stucky, G. D. Science 2000, 287, 465-467.

(10) Chen, J .; Weimer, W. A. J . Am. Chem. Soc. 2002, 124, 758-759.

(11) Delamarche, E.; Bernard, A.; Schmid, H.; Michel, B.; Biebuyck,

H. Science 1997, 276, 779-781.

(12) Delamarche, E.; Bernard, A.; Schmid, H.; Bietsch, A.; Michel, B.; Biebuyck, H. J . Am. Chem. Soc. 1998, 120, 500-508.

(13) Papra, A.; Bernard, A.; J uncker, D.; Larsen, N. B.; Michel, B.; Delamarche, E. Langmuir 2001, 17, 4090-4095.

(14) Xiao, D.; Zhang, H.; Wirth M. Langmuir 2002, 18, 9971-9976.

(15) Myers, D. Surfaces, Interfaces and Colloids; Wiley-VCH Publishers: New York, 1999.

(16) Xia, Y.; Tien, J .; Qin, D.; Whitesides, G. M. Langmuir 1996, 12 , 4033-4038

(17) Ferguson, G. S.; Chaudhury, M. K.; Biebuyck, H. A.; Whitesides, G. M. Macromolecules 1993, 26, 5870-5875.
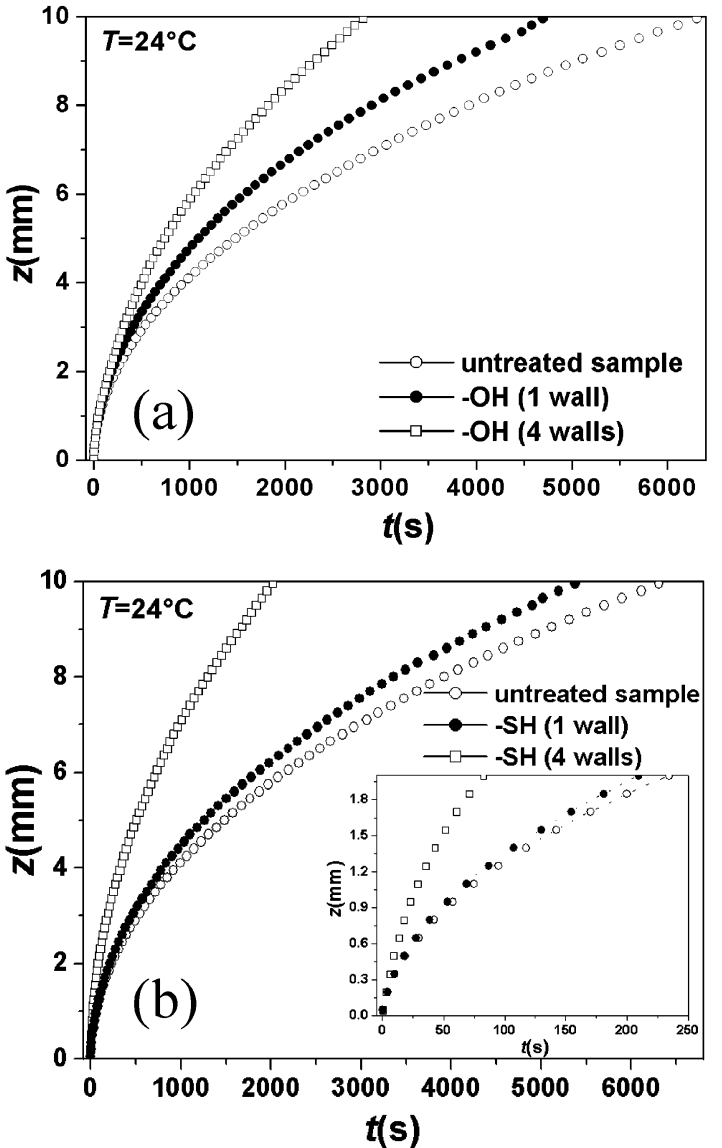

Figure 3. Experimental ( $\mathrm{z}, \mathrm{t})$ curves describing the capillary motions into untreated $\mu$-channels (empty circles), functionalized Si and untreated PDMS (full circles), and completely functionalized $\mu$-channels (empty squares), for $-\mathrm{OH}$ (a) and - SH (b) terminations, respectively. The inset shows the zoom of the region, $z<2 \mathrm{~mm}$. For clarity, we showed only one-third of the collected experimental points.

are displayed in Figure 3a. The time to completely fill the capillaries was decreased by a factor 1.3 by using hydroxylated supports with untreated replicas and by over a factor of 2 by employing four $-\mathrm{OH}$ terminated walls.

Better results were obtained by $-\mathrm{SH}$ functionalities. These were linked to the previously $-\mathrm{OH}$ terminated surfaces by adsorption of (3-mercaptopropyl)trimethoxysilane (3-MPS) from ethanol solutions (step 2 in Figure 2). Indeed, as described by Whitesides and co-workers, the silica-like layer (PDMSOX), resulting from exposing PDMS to an $\mathrm{O}_{2}$ plasma, can be employed as base layer to adsorb different silanes with various polar groups, $\mathrm{X}$ (PDMS $\left.{ }^{\circ} / \mathrm{O}_{3} \mathrm{SiX}\right) .{ }^{17-19}$ Different methods have been previously suggested to chemically modify the PDMS surface, including the self-assembly, possibly mechanically assisted, ${ }^{20}$ of monolayers from vapor phase, ${ }^{17-19}$ incubation, ${ }^{21}$ and atom-transfer radical polymerization. ${ }^{14}$ To achieve - SH functionalized PDMS (PDMS ${ }^{\mathrm{SH}}$ ), we chose functionalization from the liquid phase, which offers higher stability with respect to the ambient conditions, i.e., humidity and atmosphere. Using - SH terminated Si substrates slightly improved the MIMIC performances

(18) Ferguson, G. S.; Chaudhury, M. K.; Sigal, G. B.; Whitesides, G. M. Science 1991, 253, 776-778.

(19) Chaudhury, M. K.; Whitesides, G. M. Science 1992, 256, 12301232

(20) Genzer, J .; Efimenko, K. Science 2000, 290, 2130-2133.

(21) Böhme, P.; Vedantham, G.; Przybycien, T.; Belfort, G. Langmuir $1999,15,5323-5328$ 
(improvement factor $\cong 1.1$ ), whereas PDMSSH allows a decrease in the filling time of the $\mu$-channels by over a factor of 3 (Figure 3b).

Besides thedirect influence on the PDMS-fluid contact angle, several possible mechanisms can favor the penetration of polyurethanewithin PDMS ${ }^{\mathrm{SH}}$ toa larger extent than PDMSOx, such as the presence of hydrophobic residues contaminating the PDMS after the plasma exposure, and the adsorption of molecules of the filling liquid ontothesilica-likelayer of PDMS ${ }^{\circ x}$. Thesecan slow the polymer motion in the capillaries and can be eased or removed by the wet treatment leading to $-\mathrm{SH}$ functionalized PDMS and by the presence of 3-MPS on PDMSSH.

A further increase of thethroughput of themicrofluidics was achieved by exploiting both the - $\mathrm{SH}$ assisted liquid penetration and the strong temperature dependence of the viscosity, $\eta$, of the filling glass forming liquid. The temperature dependence of the penetration speed, implicit in the MIMIC rate law, is mainly due to $\eta$. The dramatic increase of the molecular mobility of the filling liquid as the temperature increases causes the abrupt decrease of the structural characteristic relaxation time and the viscosity. The temperature dependence of $\eta$ for glassforming liquids is usually well-described by a VogelFulcher-Tamman law (VFT): ${ }^{22} \eta(\mathrm{T})=\eta_{0} \exp (\mathrm{DT} d$ $\left(T-T_{0}\right)$ ), where $D$ is referred toas thestrength parameter and $T_{0}$ is thetemperature for which the divergence in the polymer structural relaxation time and, consequently, in the viscosity, is predicted. D determines the fragility of the compound: ${ }^{23} \mathrm{~A}$ larger fragility (smaller D) indicates a more sudden decrease of the characteristic time scale of the diffusive motions as temperature increased. Consequently fragile liquids are more suitable for highthroughput hot lithographies. We previously estimated for our filling polymer $D$ values ${ }^{24}$ which aretypical of quite fragile fluids.

The $(z, t)$ and $(v, t)$ curves obtained by increasing the temperatureareshown in Figure 4. A penetration process 2.3 times faster than that at room temperature was observed at $60{ }^{\circ} \mathrm{C}$ in untreated surfaces. The filling time instead decreased by almost an order of magnitude by employing - SH terminated capillary walls at high temperature. All the collected experimental curves are fitted by the MIMIC rate equation (fitting lines in Figure 4a), according to which

$$
z=A t^{1 / 2}
$$

where $A \approx(\Delta \mathrm{p} / \eta)^{1 / 2}$ and $\Delta \mathrm{p}$ is the effective pressure difference driving the capillary motion. In our experi-

(22) Ngai, K. L.; Plazek, D. J . Physical Properties of Polymers Handbook; Mark,. J. E., Ed.; American Institute of Physics Press: Woodbury, NY, 1996; pp 341-362.

(23) Bohmer, R.; Angell, C. A. Phys. Rev. B 1992, 45, 10091-10094.

(24) Pisignano, D.; Sariconi, E.; Mazzeo, M.; Gigli, G.; Cingolani, R. Adv. Mater. 2002, 21, 1565-1567.

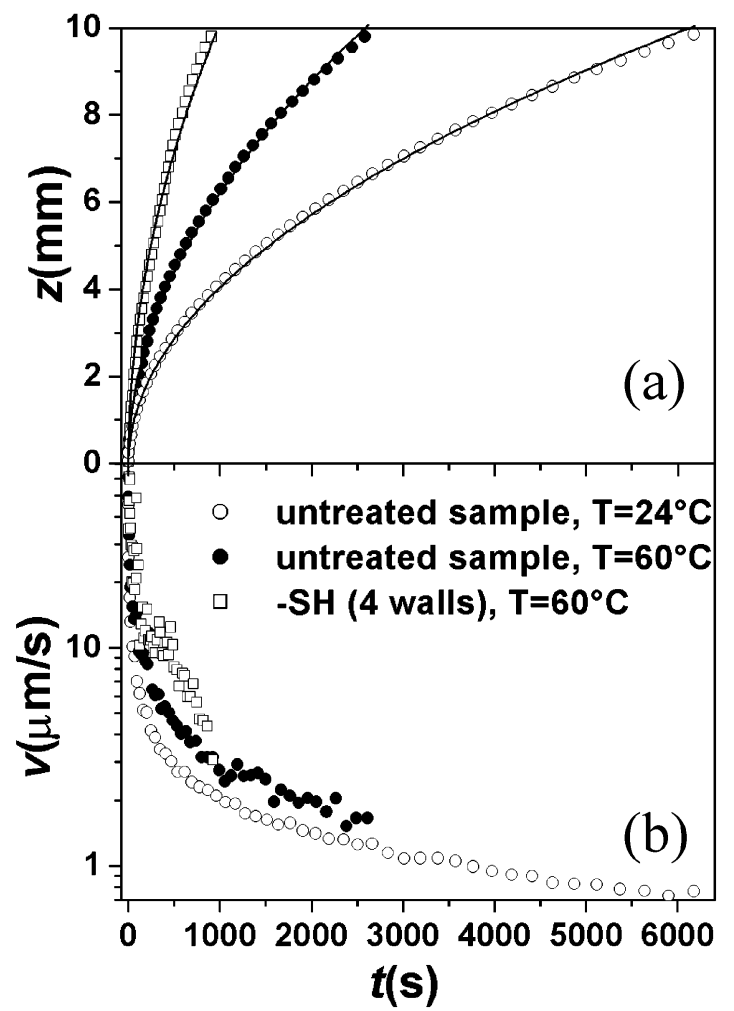

Figure 4. Experimental $(\mathrm{z}, \mathrm{t})(\mathrm{a})$ and $(\mathrm{v}, \mathrm{t})(\mathrm{b})$ curves for the capillary motion intountreated $u$-channels at room temperature (empty circles) and at $60^{\circ} \mathrm{C}$ (full circles) and into $\mu$-channels with four $-\mathrm{SH}$ terminated walls at $60^{\circ} \mathrm{C}$ (empty squares) $(25 \%$ of the collected data points shown). The solid curves superimposed to experimental $(z, t)$ data are the best fit to eq 1 .

ments at room temperature, $\Delta p$ is enhanced by a factor around 3.0 by the $-\mathrm{SH}$ functionalization $[\Delta \mathrm{p}(\mathrm{T},-\mathrm{SH})=$ $\left.(\mathrm{A}(\mathrm{T},-\mathrm{SH}) / \mathrm{A}(\mathrm{T}))^{2} \Delta \mathrm{p}(\mathrm{T})\right]$. Interestingly, only a slightly smaller factor (2.6) was found for the-SH induced increase of the effective driving pressure at $60^{\circ} \mathrm{C}$, which clearly indicates the good thermal stability of the layer at high temperatures.

We underline that, notwithstanding the wide number of well-established results about self-assembled monolayers onto inorganic supports, ${ }^{1}$ the investigation of the molecular adsorption to tailor the physi cochemical properties of polymer surfaces is a very recent research area (ref 21 and references therein). Using organic modifiers of the physicochemical properties of surfaces involved in MIMIC opens the way to new possible applications of customized molecular self-assembly, being an excellent example of how bottom-up approaches can effectively contributetoimprovetheperformances of inherently topdown lithographies.

LA049943Q 